\title{
EPITAXIAL LATERAL OVERGROWTH OF GaN WITH CHLORIDE-BASED GROWTH CHEMISTRIES IN BOTH HYDRIDE AND METALORGANIC VAPOR PHASE EPITAXY
}

\author{
R. Zhang, ${ }^{\mathrm{a}, \mathrm{b}}$, L. Zhang ${ }^{\mathrm{a}}$, D.M. Hansen ${ }^{\mathrm{a}}$, Marek P. Boleslawski ${ }^{\mathrm{c}}$, K.L. Chen ${ }^{\mathrm{b}}$, D.Q. Lu ${ }^{\mathrm{b}}$, B. Shen ${ }^{\mathrm{b}}$ \\ Y.D. Zheng ${ }^{\mathrm{b}}$, and T.F. Kuech ${ }^{\mathrm{a}}$ \\ ${ }^{a}$ Department of Chemical Engineering, University of Wisconsin, Madison, WI 53706 \\ ${ }^{\mathrm{b}}$ Department of Physics, Nanjing University, Nanjing 210093, China \\ ${ }^{c}$ Aldrich Chemical, Milwaukee, WI
}

\section{Cite this article as: MRS Internet J. Nitride Semicond. Res. 4S1, G4.7 (1999)}

\begin{abstract}
Epitaxial lateral overgrowth (ELO) of $\mathrm{GaN}$ on $\mathrm{SiO}_{2}$-masked (0001) $\mathrm{GaN}$ substrates has been investigated by using chloride-based growth chemistries via hydride vapor phase epitaxy (HVPE) and metal organic vapor phase epitaxy (MOVPE). Diethyl gallium chloride, $\left(\mathrm{C}_{2} \mathrm{H}_{5}\right)_{2} \mathrm{GaCl}$, was used in as the MOVPE Ga precursor. The lateral and vertical growth rates as well as the overgrowth morphology of ELO GaN structures are dependent on growth temperature, V/III ratio and the in-plane orientation of the mask opening. A high growth temperature and low V/III ratio increase the lateral growth rate and produce ELO structures with a planar surface to the GaN prisms. High-quality coalesced and planar ELO GaN has been fabricated by both growth chemistries. The use of the diethyl gallium chloride source allows for the benefits of HVPE growth to be realized within the MOVPE growth environment.
\end{abstract}

\section{INTRODUCTION}

$\mathrm{GaN}$ and related compounds are being developed for short wavelength light-emitting devices, such as laser (LD) and light-emitting diodes (LED), in addition to high temperature and high power electronics. GaN-based LEDs and LDs have been successfully developed and are being commercialized. Several factors impede the further development of $\mathrm{GaN}$ devices. A principal difficulty is the high density of dislocations within the GaN epilayers, which can be as high as $10^{8}-10^{10} / \mathrm{cm}^{2}$ [1]. Recently, epitaxial lateral overgrowth (ELO) has been demonstrated to effectively reduce the dislocation density in the GaN epilayers within the lateral overgrown regions [2]. Long-lifetime GaN LDs fabricated on ELO-grown materials has been reported [3]. Most reports on GaN ELO have used the metalorganic vapor phase epitaxy (MOVPE) growth technique [4,5]. Hydride vapor phase epitaxy (HVPE) is also a very attractive technique for GaN ELO. HVPE utilizes $\mathrm{GaCl}$ generated in situ through the reaction of liquid $\mathrm{Ga}$ with $\mathrm{HCl}$. HVPE offers a high growth rate and high material quality for GaN growth [6,7]. The typical growth rate can be as high as $100-200 \mu \mathrm{m} / \mathrm{hr}$. The halide process has been shown to offer a higher lateral-tovertical growth rate ratio [8] than MOVPE materials, which is critical for ELO. GaN produced by the HVPE technique does result in a greatly reduced intensity of the defect-based luminescence referred to as the yellow band (YL) when compared to the TMG-based MOVPE materials. This has been attributed to the lack of carbon in the HVPE growth system but is present in the growth sources used in MOVPE-growth systems. Intentional carbon introduction 
into HVPE materials can result in the appearance of YL [9]. If a controlled amount of $\mathrm{GaCl}$ can be introduced into a cold wall MOVPE reactor, a low carbon source could be combined with the advantages of the MOVPE reactor environment. Diethyl gallium chloride (DEGaCl) is used here as a $\mathrm{Ga}$ source in $\mathrm{GaN} \mathrm{ELO}$ growth. $\mathrm{DEGaCl}$ will decompose to $\mathrm{GaCl}$ through the $\beta$-elimination reaction in the gas phase allowing the in situ formation of $\mathrm{GaCl}$ at relatively low gas phase temperatures:

$$
\left(\mathrm{C}_{2} \mathrm{H}_{5}\right)_{2} \mathrm{GaCl} \rightarrow \mathrm{GaCl}+2 \mathrm{C}_{2} \mathrm{H}_{4}+\mathrm{H}_{2} \quad \text { (1) }
$$

The decomposition of the $\mathrm{DEGaCl}$ to $\mathrm{GaCl}$ near the growth front makes it possible to realize a high quality growth of GaN through a HVPE chemistry at high V/III ratio. This source was successfully used in GaAs growth that resulted in high uniformity, low carbon content GaAs film $[10,11]$. GaAs selective area growth, with a complete selectivity of GaAs growth with respect to $\mathrm{SiO}_{2}, \mathrm{Si}_{3} \mathrm{~N}_{4}$, and $\mathrm{Al}_{2} \mathrm{O}_{3}$ masking materials, has been demonstrated over a wide range of process window $[12,13,14]$. The present work demonstrates the utility of the DEGaCl source in GaN ELO. In this paper, we systematically investigate the GaN ELO by using both HVPE and MOVPE with DEGaCl as the Ga source. It has been found that both the growth rate and the geometric shape of the GaN prisms are dependent on the growth condition and the orientation of the window opening. High growth temperatures and a low V/III ratio are helpful in enhancing the lateral overgrowth. Under optimized growth conditions, high quality ELO GaN films are produced with a planar surfaces and free of observable voids at the coalescence interface.

\section{EXPERIMENTS AND RESULTS}

The initial GaN 'substrate' is a $1 \mu \mathrm{m}$ thick GaN film grown by MOVPE on a (0001) sapphire substrate. The masking material a $\sim 100 \mathrm{~nm}$ thick patterned $\mathrm{CVD} \mathrm{SiO}_{2}$ layer. There are two kinds of pattern used in this study. The first pattern is a radial pattern consisting of many $\sim 5 \mu$ wide stripe openings in the masking materials with a $0.74^{\circ}$ angle separation. An additional pattern consisted of $2-4 \mu \mathrm{m}$ wide parallel stripe openings on a $12 \mu \mathrm{m}$ pitch oriented along the $<1 \overline{1} 00>$ orientation. The substrates were used in a subsequent ELO GaN growth step in both HVPE and MOVPE systems, with DEGaCl as the Ga source in the latter case. In the HVPE process, the growth temperature is varied over the range of $1030-1100^{\circ} \mathrm{C}$. The input V/III ratio, calculated as the ratio of the input $\mathrm{NH}_{3}$ to input $\mathrm{HCl}$ flow rates, is controlled between 33 to 83 corresponding to a $\mathrm{NH}_{3}$ mole fraction, $\left[\mathrm{NH}_{3}\right]$, of $0.076-0.12$. Nitrogen was used as the carrier gas. In the MOVPE process, the growth temperature is varied over the range of $1000-1100^{\circ} \mathrm{C}$, while the input V/III ratio is varied over the range of 1800-3400. The hydrogen is used as the carrier gas in this case.

The extent and properties of the GaN ELO on $\mathrm{SiO}_{2}$-masked substrates were determined. Both the growth rate and the morphology of ELO regions depend on the growth conditions and stripe or opening orientation. Figure 1 presents the typical growth morphology, obtained through scanning electron microscopy (SEM) on a HVPE GaN ELO sample on a radial patterned substrate using the growth conditions of $\mathrm{T}_{\mathrm{g}}=1050^{\circ} \mathrm{C}$, input mole fraction $\left[\mathrm{NH}_{3}\right]=0.12$ and $\mathrm{HCl}$ input mole fraction of $[\mathrm{HCl}]=0.0022$. In the Figure 1 (a) is an image of an ELO GaN prism obtained within an opening oriented along the $<11 \overline{2} 0>$ direction while Figure 1(c) is an image of a $<1 \overline{1} 00>$-oriented prism. The prism shown in Figure 1(b) was formed in a direction intermediate to the $<11 \overline{2} 0>$ and $<1 \overline{1} 00>$ directions. The prism cross-section changes with the 


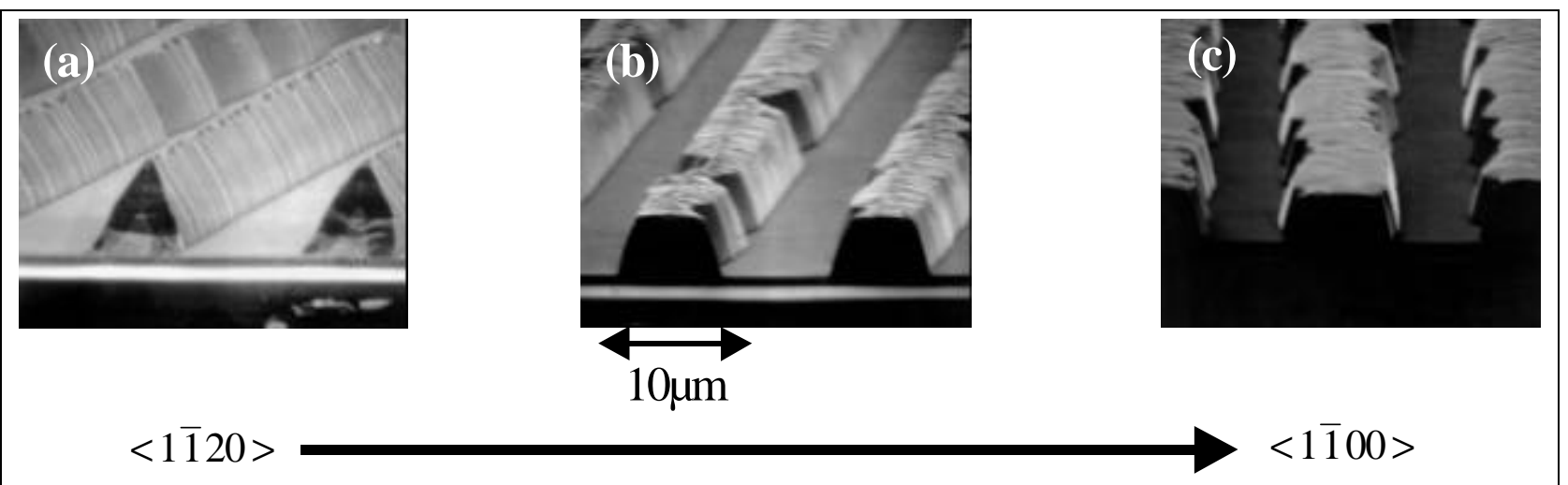

Figure 1: The orientation dependence of GaN ELO prisms grown by HVPE. The stripe opening was varied from the $<11 \overline{2} 0>$ to $<1 \overline{1} 00>$ in-plane directions.

different stripe orientations. The cross-section of the $\langle 11 \overline{2} 0>$ prisms is a triangle with a base angle of $\sim 62^{\circ}$, which indicates that these prism sidewalls are (1 $\left.\overline{1} 01\right)$ planes. The cross-sectional shape of the $<1 \overline{1} 00>$-oriented prism is a trapezoid. It is worthwhile to point out that the $<11 \overline{2} 0>$-oriented prism has the smoothest sidewalls, while the $<1 \overline{1} 00>$-oriented prism has much rougher sidewalls. This may indicate that the (1 $\overline{1} 01)$ planes are the more stable growth facets under these growth conditions. Both the lateral and vertical growth rates are dependent on the stripe opening orientation. From Figure 1, we find that the $<11 \overline{2} 0>$-oriented prism has the lowest lateral growth rate but the highest vertical growth rate. The $<1 \overline{1} 00>$-oriented prism has the highest lateral growth rate and the lowest vertical growth rate.

The SEM observations also reveal a strong dependence of the geometric prism shape on the specific growth conditions. Figure 2 shows two SEM micrographs of the cross-sections of GaN HVPE ELO prisms oriented along the $<11 \overline{2} 0>$ and $<1 \overline{1} 00>$ directions, respectively. The growth conditions employed for these samples were $\mathrm{T}_{\mathrm{g}}=1100^{\circ} \mathrm{C},\left[\mathrm{NH}_{3}\right]=0.076$ and $[\mathrm{HCl}]=0.023$. The conversion efficiency of the $\mathrm{HCl}$ to $\mathrm{GaCl}$ is approximately 60-70\%. As the growth temperature is elevated and the V/III ratio decreased, the cross-sectional shape of the ELO prisms change. The $\langle 11 \overline{2} 0>$ prism cross-section changes from a triangle to a rectangle, and that of the $<1 \overline{1} 00>$ prism changes from a trapezoid to a reverse-trapezoidal structure. The latter result has not been previously reported in the GaN ELO literature.

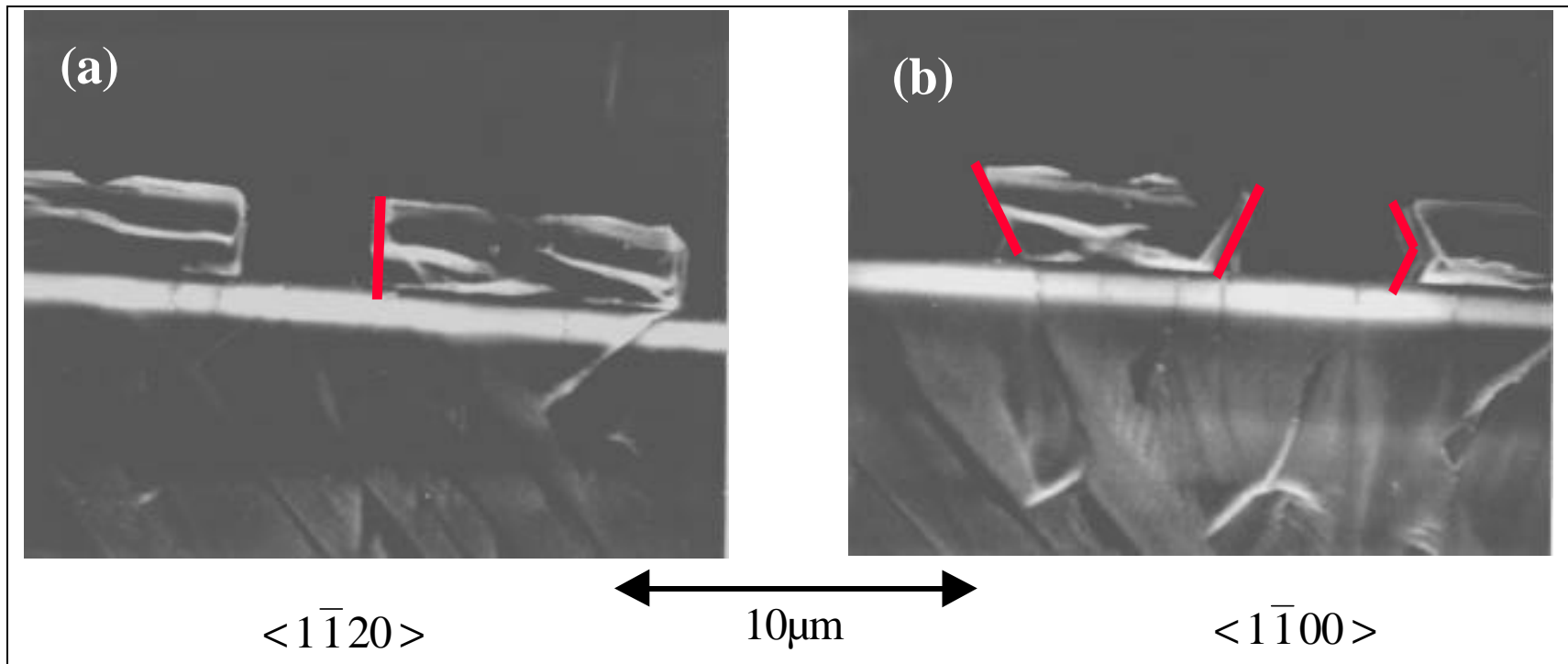

Figure 2. The orientation dependence of cross-sections of prisms grown under high temperature 


\section{$<1 \overline{1} 00>$

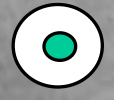

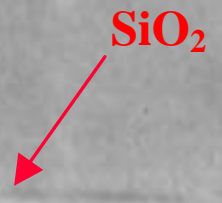

\section{$10 \mu \mathrm{m}$}

Figure 3: Cross-sectional SEM picture of a coalesced ELO GaN sample grown by HVPE. There is no void observed at the coalescence interface.

growth conditions, high-quality coalesced ELO GaN films on parallel patterned substrates, having planar surfaces, have been fabricated by the HVPE technique. A cross-sectional SEM image of the coalesced film is shown in Figure 3. The growth temperature is $1100^{\circ} \mathrm{C}$ and $\left[\mathrm{NH}_{3}\right]=0.076,[\mathrm{HCl}]=0.023$ for this sample. No observable void was found at the coalescence interface under these conditions.

MOVPE GaN ELO, when using DEGaCl, exhibit similar trends to the HVPE samples. As shown in Figure 4, the MOVPE ELO GaN samples grown in $<1 \overline{1} 00>$-oriented parallel stripes under the same V/III ratio of 3500, have a changing morphology with growth temperature. The cross-section of ELO GaN prisms is strongly dependent on growth temperature over the range $1100^{\circ} \mathrm{C}$ to $1000^{\circ} \mathrm{C}$. The cross-section of ELO GaN prisms grown at $1100^{\circ} \mathrm{C}$ is close to rectangular, while those of $\mathrm{ELO} \mathrm{GaN} \mathrm{prisms} \mathrm{grown} \mathrm{at} \mathrm{lower} \mathrm{than} 1050^{\circ} \mathrm{C}$ are triangular. The lateral growth rate of ELO GaN increases with increasing growth temperature while the growth temperature at this V/III ratio only weakly influences the vertical growth rate.

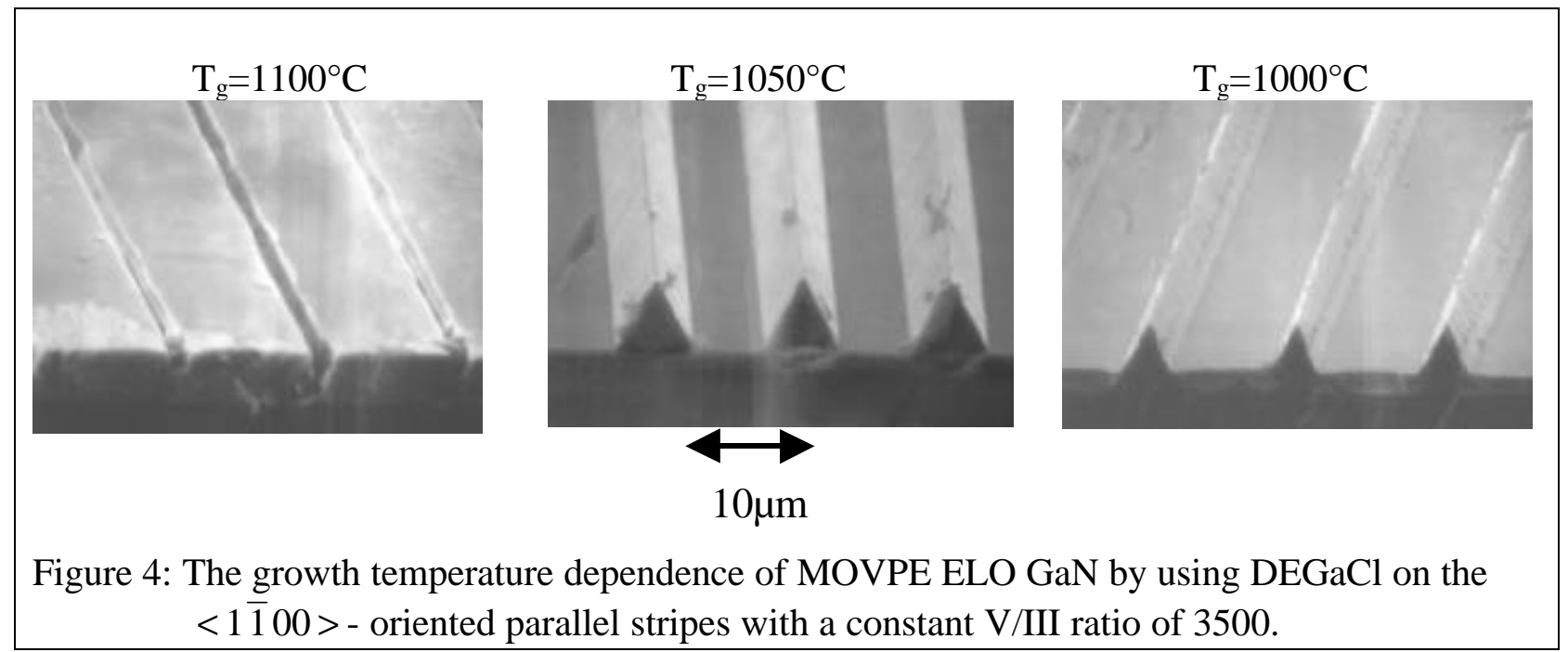

The dependence of MOVPE GaN ELO, using DEGaCl, on the V/III ratio during growth has been investigated and is shown in Figure 5. These GaN ELO samples were grown at the growth temperature of $1100^{\circ} \mathrm{C}$ over the V/III ratio range of 3500-1800. The cross-section of ELO GaN prisms grown with V/III ratio of 1800 is rectangular, while those of ELO GaN prisms grown with higher V/III ratio are trapezoidal, with sloping facets becoming evident. The lateral and vertical growth rates are somewhat dependent on the V/III ratio at this temperature with a lower V/III ratio leading to a higher vertical growth rate. 


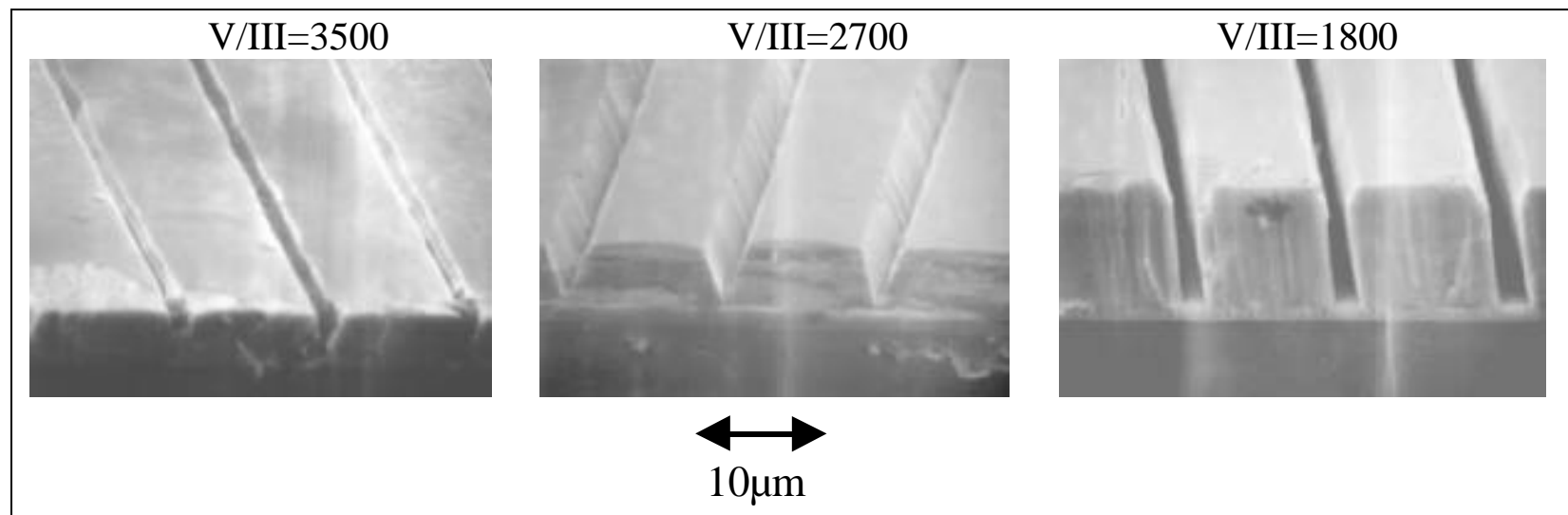

Figure 4: The dependence of MOVPE ELO GaN by using DEGaCl with the $<1 \overline{1} 00>$-oriented parallel stripes pattern at the constant growth temperature of $1100^{\circ} \mathrm{C}$.

\section{DISCUSSION}

The resulting structure during the ELO process is known to be dependent on the gas phase transport of reactants to the growth front, which is dependent on the growth conditions and mask geometry. The difference in growth behavior between the inorganic, GaCl-based growth, and conventional MOVPE ELO using trimethyl gallium, also indicates that the growth chemistry plays an important role in the local growth rates and facet formation [8]. The chemical and physical properties of mask and substrate materials affect the surface diffusion [15], nucleation and kinetics. The lateral and vertical growth rates of ELO GaN are thus determined by the interplay of these various influences at the growth front. The local growth environments near a mask opening will differ from those in large-scale deposition and the optimization of the material properties and growth habit of the localized regions may be quite different from that found on non-masked regions. Under the conventional conditions for HVPE deposition, it has been suggested from the observed large surface roughness of HVPE-grown large scale GaN films that the (0001) plane is not the most stable growth facet [6]. Under such conditions, the most stable growth front is thought to be the $(1 \overline{1} 01)$ planes. Higher growth temperatures and lower V/III ratios decrease the supersaturation (driving force) [8]. The resulting lower growth rate would enhance the surface diffusion rate of reactants over the (0001) plane to adjacent, more favored growth facets and result in a higher lateral growth rate and a variety of prismatic structures. The use of a chloride-containing Ga source in both these systems allows for the addition of several chemical reactions to the growth front chemistry. The simplest set of chemical reactions important at the $\mathrm{GaN}$ growth front in the case of chloride-based growth is:

$$
\begin{aligned}
\mathrm{GaCl}+\mathrm{NH}_{3} & \rightarrow \mathrm{GaN}+\mathrm{HCl}+\mathrm{H}_{2} \\
\mathrm{GaN}+\mathrm{HCl} & \Leftrightarrow \mathrm{GaCl}+\frac{1}{2} \mathrm{~N}_{2}+\frac{1}{2} \mathrm{H}_{2}
\end{aligned}
$$

where in the $\mathrm{DEGaCl}$ case, the $\mathrm{GaCl}$ is generated through the intramolecular decomposition of the growth source as presented in Equation 1. Reaction 3 implies equilibrium between the steady state concentrations of $\mathrm{HCl}$ and $\mathrm{GaCl}$ at the growth front. The MOVPE growth front has often been considered to be near thermodynamic equilibrium [16]. If this is the case for the high temperature growth of $\mathrm{GaN}$, the supersaturation at the growth front will decrease with increasing temperature due the reversible reaction associated with the $\mathrm{HCl}$ reacting at the $\mathrm{GaN}$ surface. A similarity between the growth habits and behavior between the HVPE and MOVPE-DEGaCl systems is therefore expected.

The presence of chlorine in the growth system can modify the growth habit from the trimethyl gallium based growth. Experimentally, the chloride-based systems lead to both higher lateral-to-vertical growth and desirable growth facets, such as vertical sidewalls over a broader range of growth conditions. The near surface concentration of $\mathrm{GaCl}$ in the HVPE system is 
higher than in the $\mathrm{DEGaCl}$ systems. If adsorbed chlorine or $\mathrm{GaCl}$ species can stabilize certain facets, the higher concentration of $\mathrm{GaCl}$ within the HVPE would more readily promote the formation of specific facets, such as the $\{1 \overline{1} 00\}$ planes, over the DEGaCl-based MOVPE growth. Regardless of the specific chemical mechanism, the use of $\mathrm{GaCl}$ within either of these systems leads to improved ELO characteristics over conventional MOVPE growth using simple metal alkyls.

\section{CONCLUSIONS}

ELO of GaN has been systematically investigated by using chloride-based growth chemistry in both HVPE and MOVPE, through the use of DEGaCl, systems. The lateral and vertical growth rates as well as the morphology of the ELO regions depend on the growth condition and orientations of opening stripes. The high growth temperatures and a low V/III ratio enhance the lateral overgrowth. The reverse trapezoid prism has been fabricated in [1 100$]$ stripes under the growth temperature of $1100^{\circ} \mathrm{C}$ and the V/III ratio of 33. Under the optimized ELO conditions, high quality flat-topped and coalesced GaN ELO films have been successfully fabricated and no observable voids found at the coalescence interface.

\section{ACKNOWLEDGEMENTS}

R. Z., K.L. Chen, D.Q. Lu, B. Shen and Y.D. Zheng thank the financial support of the China "863" national high-tech development program. The authors would like to acknowledge the financial support of the ONR MURI on Compliant Substrates, and the facilities support of the NSF Materials Research Science and Engineering Center on Nanostructured Materials and Interfaces, USA.

\section{REFERENCES}

1 F.A. Ponce and D.P. Bour, Nature, 386, 351(1997).

2 A. Sakai, H. Sunakawa and A. Usui, Appl. Phys. Lett., 71, 2259(1997).

3 S. Nakamura, M. Senoh, S. Nagahama, N. Iwase, T. Yamada, T. Matsushita, H. Kiyoku, Y. Sugimoto, T. Kozaki, H. Umemoto, M. Sano and K. Chocho, Appl. Phys. Lett., 72, 211(1998).

4 O-H. Nam, M.D. Bremser, T.S. Zheleva and R.F. Davis, Appl. Phys. Lett., 71, 2638(1997)

5 H. Matsushima, M. Yamaguchi, K. Hiramatsu and Sawaki, Proc. $2^{\text {nd }}$ Int. Conf. Nitride Semiconductors, Tokushima, Japan, 1997, p492.

6 N.R. Perkins, M.N. Horton, Z.Z. Bandic, T.C. McGill and T.F. Kuech, Mat. Res. Sci. Symp. Proc. 395, 243 (1996).

7 R.J. Molnar, K.B. Nichols, P. Maki, E.R. Brown and I. Melngailis, Mater. Res. Soc. Symp. Proc., 378, 479 (1995).

8 J-O Carlsson, Solid State \& Mater. Sci., 16, 161(1990).

9 R. Zhang and T.F. Kuech, Appl. Phys. Lett. 72, 1611-1613 (1997).

10 T. F. Kuech, R. Potemski and F. Cardone J. Cryst. Growth V124, 318-325 (1992).

11 A. Narmann, M.L.Yu Surface Science V270, 1041-1047 (1992).

12 T.F. Kuech, J. Cryst. Growth V115, 52-60 (1991).

13 Ko-ichi Yamaguchi, K. Okamoto, Jpn. J. Appl. Phys. V32 4885-4888, (1993).

14 Y. Shiraishi, N. Furuhata, A. Okamoto, J. Cryst. Growth V182, 255-265 (1997).

15 A. Usui and T. Nishinaga, Jpn. J. Appl. Phys., 36, L899(1997).

16 G.B. Stringfellow, Organometallic Vapor Phase Epitaxy, (Academic Press, San Diego, 1989) Chap. 3. 